\title{
Chronic Recurrent Non-specific Parotitis: A Case Report and Review
}

\author{
Saibaba Mahalakshmi ${ }^{1}$, Srinivas Kandula ${ }^{2}$, Patil Shilpa ${ }^{3}$, Ganganna Kokila ${ }^{4 *}$
}

OPEN ACCESS

Citation: Saibaba Mahalakshmi, Srinivas Kandula2, Patil Shilpa, Ganganna Kokila. Chronic Recurrent Non-specific Parotitis: A Case Report and Review. Ethiop J Health Sci 2017;27(1):95-100. doi: 13

Received: June 7, 2016

Accepted: August 29, 2016

Published: January 1, 2017

Copyright: Saibaba M, et al. This is an open access article distributed under the terms of the Creative Commons

Attribution License, which permits unrestricted use, distribution, and reproduction in any medium, provided the original author and source are credited.

Funding: Nil

Competing Interests: The authors

declare that this manuscript was approved

by all authors in its form and that no

competing interest exists.

Affiliation and Correspondence:

${ }^{1}$ Department of Oral Medicine and

Radiology, Sri Siddhartha Dental

College \& Hospital, Sri Siddhartha

Academy of Higher Education,

Tumkur

${ }^{2}$ Department of Oral Medicine and Radiology, Kalinga Institute of Dental Science, KIIT University, Bhubneshwar

3Department of Oral Medicine and Radiology, AECS Maaruti College of Dental Sciences \& Research Centre, Bangalore

4Department of Oral Pathology and Microbiology, Sri Siddhartha Dental College \& Hospital, Sri Siddhartha Academy of Higher Education, Tumkur

*Email: ammisaiganesh@gmail.com

\section{ABSTRACT}

BACKGROUND: Chronic recurrent non-specific parotitis is characterized by recurrent episodes of swelling and pain of unknown etiology in the parotid gland. Sialography is a hallmark in the diagnosis of salivary gland disorders; newer imaging modalities like CT-Sialography, sialoendoscopy and MRI can be used. Various treatment modalities have been tried, from conservative approach to surgical excision depending on the recurrence rate and severity of the condition. Although symptomatic treatment with antibiotics and analgesic, injection of intraductal medicament, aggressive treatment like duct ligation or excision of gland are some of the treatment modalities, there is no established algorithm as to which treatment method should be opted in such clinical situation.

CASE DETAIL: A 20 years old male patient reported with pain and salty taste in the mouth that had began before a week. Examination revealed an elevated right parotid papilla; ropy, cloudy appearing saliva was oozing out on milking the gland. Unstimulated and stimulated whole salivary flow rate was assessed using drooling method. Sialography was used as a diagnostic and a therapeutic aid. In our case, sialography as a treatment showed a good response with no recurrence after two years of follow-up. We highlighted the role of sialography as a therapeutic aid.

CONCLUSION: Recurrent attacks significantly affect the quality of life and also lead to progressive gland destruction. Preventing or reducing the frequency of recurrence remains the goal of therapeutic procedure. Hence, conventional sialography is useful in the diagnosis and also effective as a therapeutic aid in recurrent parotitis.

KEYWORDS: Chronic recurrent Parotitis, Non specific Parotitis, Sialography

\section{INTRODUCTION}

Parotitis may be acute or chronic. Acute refers to sudden onset of pain and swelling in parotid gland whereas chronic recurrent parotitis (CRP) is characterized by intermittent, painful and swelling of the gland which may or may not be associated with food intake (1). The term "chronic recurrent non-specific parotitis" is used in cases where no definite etiology is identified (2). 
Etiology of CRP is unclear. Researchers have suggested that recurrent parotitis arises due to retrograde infections eventual to stasis of saliva, allergic, immune deficiency, genetic and hereditary factors $(2,3)$. However, none of these factors have been proven as an actual cause for the disease. There is lot of disagreement regarding the treatment of recurrent parotitis due to ambiguity about its etiology. Treatment of acute phase is aimed at relieving symptoms and prevention of damage to gland parenchyma (4). Proposed treatments for CRP include steroids, tetracycline and $1 \%$ methyl violet as intra-ductal medicament $(5,6)$. Conventional sialography can also be used as a therapeutic aid in cases of recurrent infection as it helps in salivary gland lavage, removal of small calculi and mucous plugs within the ducts $(7,8)$. Our primary aim in this case was to relieve the pain and reduce the frequency of recurrence. To achieve this, we used sialography as a diagnostic and therapeutic aid.

\section{CASE REPORT}

History: A 20 years old male patient presented with pain in front of the right ear and salty taste on having food that had began before a week. Pain in the right parotid gland region was sudden,

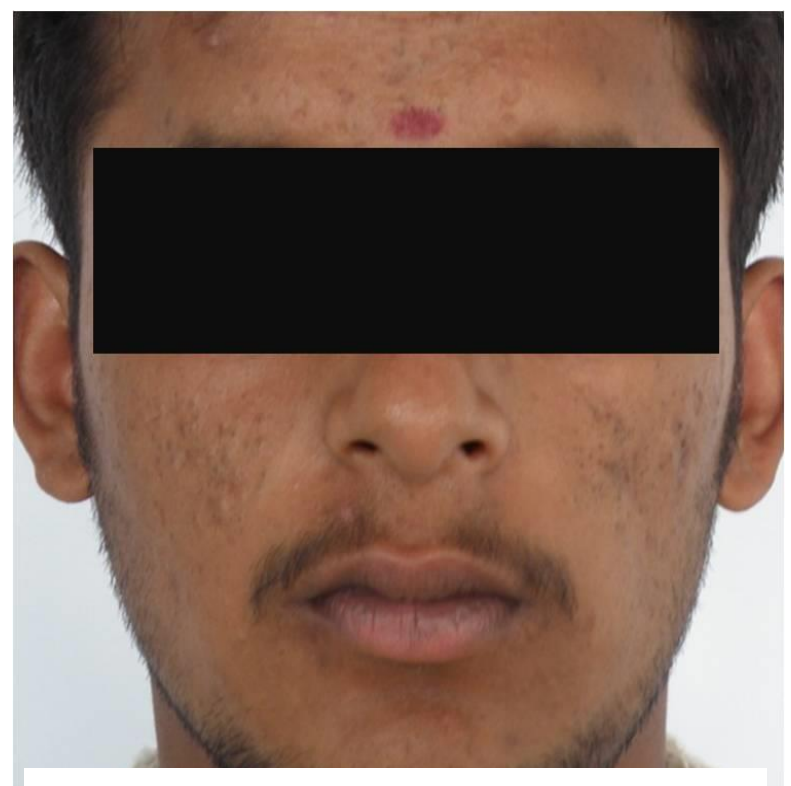

Figure 1: Extra oral view showing the bilaterally symmetrical face intermittent, throbbing in quality and initiated while the patient was chewing food and increased in intensity especially on taking citrus food. The patient gave history of at least two episodes of recurrent swelling and pain per year on the right side of the face since two-three years. The swelling and pain used to subside when the patient takes antibiotics and analgesics. His past medical history was non contributory. The patient had been smoking four cigarettes per day since six months.

Physical examination: On general physical examination, the patient was febrile and lymph nodes were palpable in the submandibular region bilaterally which was single, firm, freely mobile and tender. On extra oral examination, no gross asymmetry was seen in the face (Figure 1). Both the parotid glands were examined for tenderness and purulent discharge. The right parotid gland was tender on palpation and firm in consistency whereas the left parotid gland was normal. Intraoral examination revealed an elevated right parotid papilla (Figure 2). On milking the gland a thick, ropy, cloudy appearing saliva was oozing out of the duct. Provisional diagnosis of recurrent parotitis was then considered.

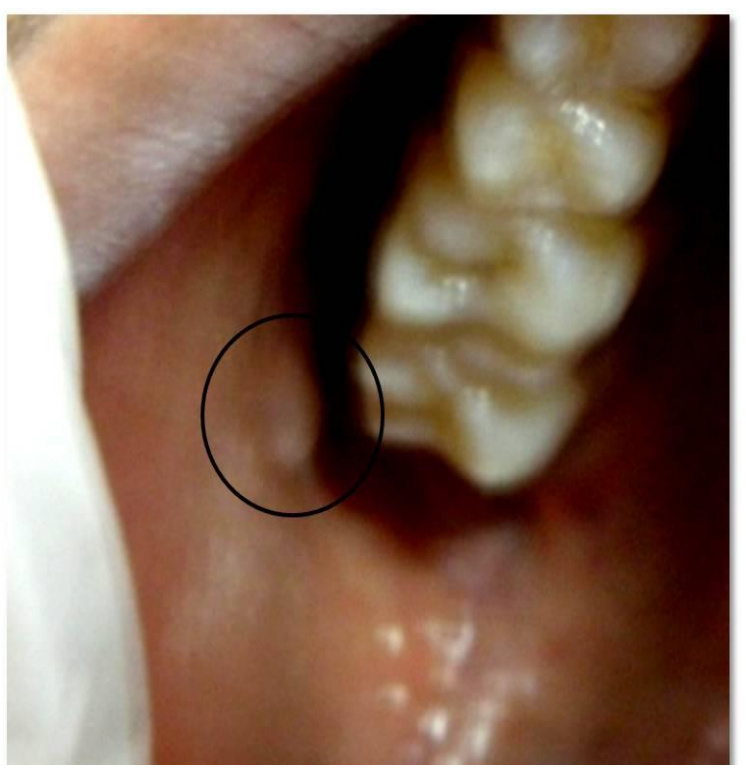

Fig ure 2: Intra oral view showing elevated right parotid papilla

DOI: http://dx.doi.org/10.4314/ejhs.v27i1.13 
Investigation: Although antibiotic sensitivity test was the norm of investigation, it was not advised as the patient was already taking antibiotic which was prescribed by a private practitioner. Salivary flow rate was assessed using drooling method. Before unstimulated whole saliva was collected,the patient was instructed to refrain from eating and drinking for 90 minutes to avoid any salivary stimulation. Later, the patient was asked to drool the saliva in a vial at every one minute for five minutes. For stimulated method, $2 \%$ citric acid was placed on the tongue at every 30 seconds for five minutes and the patient asked to drool the saliva in a vial. Salivary flow rate of unstimulated and stimulated saliva was $0.3 \mathrm{ml} / \mathrm{min}$ and $1 \mathrm{ml} / \mathrm{min}$ respectively suggesting normal salivary flow rate.

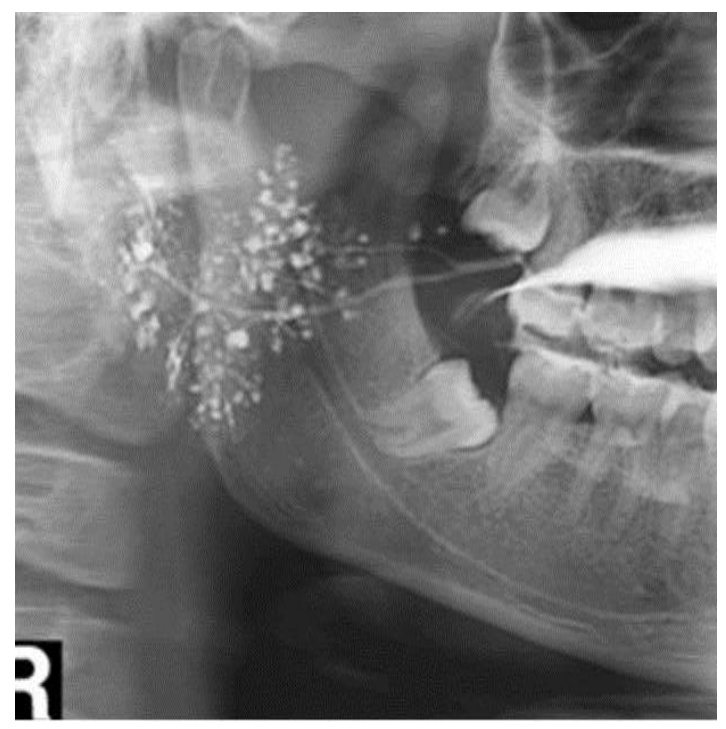

Figure 3: Sialograph shows the duct which is normal in course and caliber with dots and blobs appearance at the terminal ductules
Sialography was performed with $2 \mathrm{ml}$ of sodium diatrizoate contrast media which was slowly injected into the gland until some resistance was felt, and the patient reported slight pain in the gland area. Digital OPG showed uniform normal course and caliber of Stensen's duct measuring about 2-3 $\mathrm{mm}$ in diameter from opening till the periphery of the gland. Terminal ductules showed areas of "blobs" and "dots" of contrast media indicating Sialectasis (Figure 3). Fifty percent excretion of the dye was observed in digital OPG after one minute (Figure 4), and complete excretion of dye was observed in five minutes suggesting normal functioning of the gland. The patient was instructed to use secretogogues (lime juice) for three days to clear the debris and to stimulate the salivation.

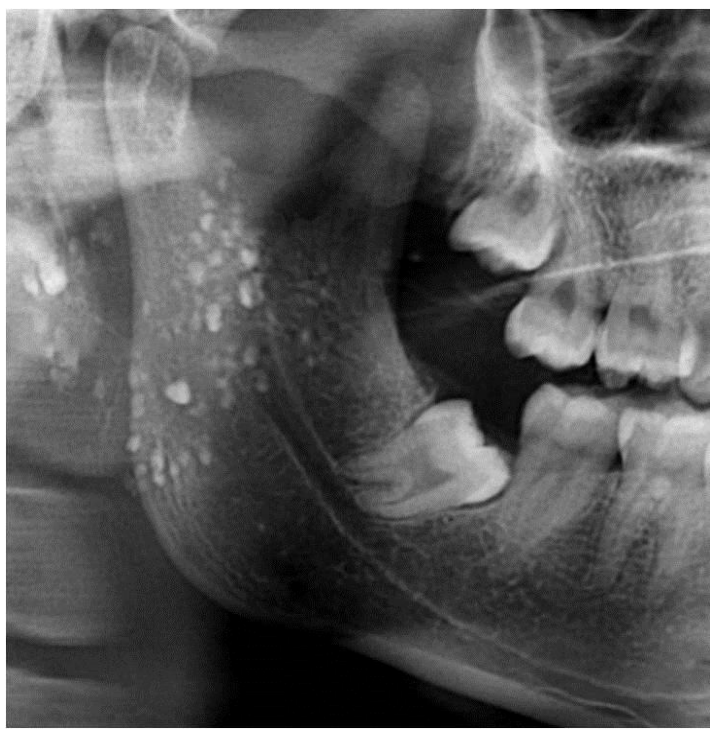

Figure 4: Approximately 50\% excretion of contrast medium after 1
Diagnosis and treatment: Based on patient history, clinical finding, investigation and 
sialographic appearance a final diagnosis of chronic recurrent non-specific parotitis was made as we were not able to find out any specific etiologic factor. Sialography was performed not only for the diagnostic purpose but also for gland lavage which helped in clearing the mucus plug or cellular debris. The patient was advised to continue antibiotics and analgesic for seven days and was kept under regular follow-up once in six months for a two years' duration. The treatment seemed to be effective as there was no recurrence during the two years' follow-up.

\section{DISCUSSION}

CRP patients suffer from recurrent swelling and tenderness of the involved gland which gradually leads to the destruction of the gland. Reducing the frequency of recurrence and improving the quality of life are the objective of treatment. Key to the successful treatment of CRP is the complete removal of cellular debris and precipitated serum proteins from the ductal lumen. This can be skillful achieved with sialography, ductal dilation with lacrimal probe and gland lavage (9).

CRP commonly occurs in middle age with female predilection. Clinical examination reveals swelling and tenderness of the involved gland. Elevated parotid papilla, reduced salivary flow and secretion are viscous and milky in appearance with clumps of material interspersed (10).

The etiology of the disease is multifactorial .There are various theories to explain the pathogenesis, One theory postulates that reduced salivary flow results in decreased mechanical cleansing, allowing bacteria to colonize and invade the duct. Retrograde infection by opportunistic oral flora can result directly in chronic recurrent sialadenitis. Whereas the other proposes that repeated episodes of acute infection may lead to mucus metaplasia of ductal epithelium resulting in increased mucus content of secretions, stasis and further episodes of inflammation. Secretory disorders like difference in the secretion and excretion of fluid are also considered as having an important role in the pathogenesis(10). Studies have reported that the ultrasonagraphy and sialography appearance for recurrent parotitis is characterized by Sialectasis with strictures and dilatation of the major duct (9). Sailoendoscopy revealed white wall and lack of vascularity in the ductal layer in $75 \%$ of CRP cases and multiple fibrinous debris and mucous plug in $45 \%$ of juvenile recurrent parotitis (11). In our case sialogram revealed Sialectasis with normal major duct caliber without any ductal pathology. Although sialography is primarily used for diagnostic purpose, it can also be used as treatment for recurrent parotitis and obstructive disorders (12). Sialography improves patency of duct during cannulation by flushing action of irrigant which helps in removing any epithelial debris and mucous plug. The Iodine content of the contrast medium acts as an antiseptic agent, thus reducing symptoms and preventing recurrence. This treatment should be repeated once in every two days along with sialogouges until the swelling is subsided and saliva is clear. In our case, Sialography was done only once and the patient showed no recurrence during the two years' follow-up, similar with many other reported cases $(5,7,8,13)$. Other intracanal medicaments have been tried by many authors-Bowling etal reported intraductal tetracycline instillation causing acinar atrophy in rats (6). Mandel and Kaynar (1995) stated that although steroid reduces swelling and inflammation, it is not effective in preventing recurrence (5).Nahileli et al. (2004) stated that use of sailoendoscopy facilitates direct visualization of the intraglandular structures and combination of steroid lavage with ductal dilatation will help in reducing the symptoms as well as recurrence(14). However, the success of the treatment depends on intraductal lavage of the affected gland rather than the type of intraductal medicament used as various studies showed no difference in the frequency of recurrence rate. Watkin and Hobsely found that $56 \%$ of adult and $64 \%$ of children showed good response to conservative treatment in a five years' follow-up study (15). Bilateral sailoendoscopy and lavage with intraductal hydrocortisone resulted in $92 \%$ recurrence free rate upto 36 months in juvenile recurrent parotitis cases (16). Few case reports showed sialography as alternative treatment for this condition as it is minimally invasive procedure with favorable outcome in juvenile recurrent parotitis (5-8). However, if the

DOI: http://dx.doi.org/10.4314/ejhs.v27i1.13 
symptom persists or worsens, then an aggressive treatment should be opted such as duct ligation, parotidectomy and tympanic neurectomy.

In conclusion, as the cause for CRP is multifactorial, patients should be educated to take higher liquid content in the diet, to do selfmassaging of the gland and to maintain oral hygiene so as to avoid retrograde infection. In spite of various advanced imaging techniques, there is no established algorithm as to which imaging modality should be done in a given clinical situation. Individual radiologist may prefer one imaging tool over another for evaluating particular problems. In our case we preferred sialography as it is simple to perform and costeffective. It also has added therapeutic effect, especially in conditions where it can prevent recurrence and provide maximum benefit to the patient.

REFERENCES
1. Nahlieli O, Bar T, Shacham R,Eliav E,Nakar LH. Management of chronic recurrent parotitis: Current therapy. J Oral Maxillofac Surg 2004;62:1150.

2. Ongole R,Praveen BN.text book of oral medicine, oral diagnosis and oral radiology, 1st ed.Elsevier publication:2010.p.348-350.

3. Reid E, Douglas., CrowY. et al, Autosomal dominant juvenile recurrent parotitis. $\mathrm{J}$ Med Genet 1998;35:417.

4. Leerdam CM, Martin HC, Isaacs D. Recurrent parotitis of childhood. J Paediatr Child Health 2005;41:631.

5. Mandel L, Kaynar A. Recurrent parotitis in children. $N$ YState Dent J 1995;61:22-5.

6. Bowling DM, Ferry G, Rauch SD, Goodman ML.Intraductal tetracycline therapy for the treatment of chronic recurrent parotitis. Ear Nose Throat J 1994;73:262-274.

7. Galili D, Marmary Y. Juvenile recurrent parotitis: Clinicoradiological follow-up study and the beneficial effect of sialography. Oral Surg Oral Med Oral Pathol 1986;61:550.

8. Chitre VV, Premchandra DJ. Recurrent parotitis. Arch Dis Child 1997;77:359-363.

9. Baurmash HD.Chronic recurrent parotitis:A Closer look at its origin, diagnosis and management. $J$ Oral Maxillofac Surg 2004:62:1010-1017.

10. Bhatty M A, Piggot TA , Soames JV,McleanNR .Chronic non-specific parotid sialadenitis. $\quad B r \quad J \quad$ Plast Surg1998 Oct;51(7):517-521.

11. Canzi P,Occhini A,Pagella,Marchal,Benazzo M.Sialoendoscopy in juvenile recurrent parotitis : a review of the literature.Acta.Otorhinolaryngol ital 2013;33:367-73.

12. Reddy SS, Rakesh N, Raghav N, Devaraju D, Bijjal SG. Sialography: Report of 3 cases. Indian J Dent Res 2009;20:499-502.

13. Hasson O. Sialoendoscopy and Sialography: Strategies for assessment and treatment of salivary gland obstructions. J Oral Maxillofac Surg 2007;65:300-4.

14. Nahlieli O, Shacham R, Shlesinger M, Eliav E. Juvenile recurrent parotitis:A new method

DOI: http://dx.doi.org/10.4314/ejhs.v27i1.13 
of diagnosis and treatment. Pediatrics 2004;114;9-12.

15. Watkin GT, Hobsley M. Natural history of patients with recurrent parotitis and punctate sialectasis. Br J Surg 1986;73:745-8.

16. Shacham R, Droma EB, London D, Bar T, Nahlieli O. Long-term experience with endoscopic diagnosis and treatment of juvenile recurrent parotitis. J Oral Maxillofac Surg 2009;67:162-7. 